\title{
DIAGNÓSTICO DA TUBERCULOSE EM UNIDADES PRISIONAIS DO OESTE PAULISTA: IMPORTÂNCIA DA CULTURA E AVALIAÇÃO DA IMPLANTAÇÃO DO TESTE RÁPIDO MOLECULAR
}

Nayara Rodrigues Monteiro, Lourdes Aparecida Zampieri D’Andrea, Paula Esteves da Silva Lima, Marta Xavier Alfredo, Mariza Menezes Romão

Centro Laboratório Regional do Instituto Adolfo Lutz de Presidente Prudente, Presidente Prudente,SP. e-mail: nrmont.bio@gmail.com

\section{RESUMO}

A Tuberculose, doença infecciosa causada por micobactérias pertencentes ao Complexo M. tuberculosis, consiste num problema de saúde pública, principalmente nas unidades prisionais cujas condições são propicias à incidência deste agravo. O objetivo deste estudo foi analisar a importância da cultura automatizada MGIT ${ }^{\circledR} 960$ BD ("padrão-ouro"), o desempenho do Teste Rápido Molecular no equipamento GeneXpert ${ }^{\circledast}$ MTB/RIF e a distribuição do quantitativo da tuberculose em cinco unidades prisionais da área de abrangência do CLR IAL PP V, no período entre outubro/2015 e outubro/2016. Foram analisadas 4.509 amostras para diagnóstico, apresentando especificidade de 99,03\%, sensibilidade de $72,73 \%$ e concordância de $97 \%$ entre os testes. Os Resultados mostraram a importância da cultura para identificação de espécie e implantação do TRM-TB na rotina laboratorial, bem como da necessidade de melhoria na qualidade e no quantitativo de amostras coletadas no sistema prisional.

Palavras-chave: tuberculose, diagnóstico, cultura, teste rápido molecular, unidade prisional.

\section{DIAGNOSIS OF TUBERCULOSIS IN PRISON UNITS OF WEST PAULISTA: IMPORTANCE OF CULTURE AND EVALUATION OF IMPLANTATION OF MOLECULAR RAPID TEST}

\begin{abstract}
Tuberculosis, an infectious disease caused by mycobacteria belonging to the $M$. tuberculosis complex, is a public health problem, especially in prisons whose conditions are conducive to the incidence of this disease. The objective of this study was to analyze the importance of the MGIT ${ }^{\circledR} 960$ BD automated culture ("gold standard"), the performance of the Rapid Molecular Test in the GeneXpert ${ }^{\circledR}$ MTB/RIF equipment and the distribution of the quantitative of tuberculosis in five prison units in the area Coverage of the RLC ALI PP $V$ in the period between October/2015 and October/2016. It were analyzed 4,509 samples for diagnosis, presenting specificity of $99.03 \%$, sensitivity of $72.73 \%$ and concordance of $97 \%$ among the tests. The results showed the importance of the culture for the identification of species and the implantation of TRM-TB in the laboratory routine, as well as the need to improve the quality and quantity of samples collected in the prison system.
\end{abstract}

Keywords: tuberculosis, diagnosis, culture, rapid molecular test, prison unit.

\section{INTRODUÇÃO}

A tuberculose é uma doença infecciosa de séria ameaça à saúde humana. O patógeno responsável por causar a infecção faz parte do gênero Mycobacterium, descrito em 1882 pelo alemão Robert Koch, conhecido como Bacilo de Koch (BK) ${ }^{1}$. O complexo M. tuberculosis (CMTB) é composto por bactérias capazes de causar a tuberculose, dentre as de maior relevância: $M$. tuberculosis, $M$. bovis, e $M$. africanum. Além das bactérias que compõem o CMTB, existem micobactérias atípicas que podem causar infecções adversas, denominadas Micobactérias não Tuberculosas (MNT) ${ }^{2,3}$.

A propagação da tuberculose está ligada às condições precárias de vida e a aglomerações de pessoas ${ }^{4}$. As unidades prisionais possuem condições favoráveis com pouca ventilação, 
superlotação gerando aglomerações que ligadas a situações sanitárias adversas e o baixo nível socioeconômico, reflete numa ameaça quanto à disseminação desse agravo ${ }^{5}$. Uma das estratégias de controle é a identificação de paciente sintomático. O diagnóstico laboratorial da tuberculose pode ser realizado através da baciloscopia, cultura e métodos moleculares. A baciloscopia trata-se da pesquisa do Bacilo Álcool Ácido Resistente (BAAR) em esfregaço biológico, corado pelo método Ziehl Neelsen. É um método simples e seguro para elucidação diagnóstica e permite a confirmação da presença do bacilo ${ }^{7,8}$.

A cultura é considerada "padrão-ouro" para o diagnóstico da tuberculose, tem alta sensibilidade e possibilita a realização de exames complementares, como a identificação da espécie ${ }^{8,10}$. O cultivo pode ser realizado em meios sólidos à base de ovos ou ágar com inibidor da microbiota contaminante. Os mais utilizados são Lowenstein Jensen (L) e Ogawa Kudoh (OK) que possibilitam a adição de substâncias como forma de isolamento de colônias, como é o caso do ácido p-nitrobenzóico (PNB) que diferencia o CMTB das outras micobactérias ${ }^{11,13}$. Podem ser utilizados também meios líquidos como o Middlebrook, principal meio de cultura dos métodos automatizados ${ }^{14}$. A cultura automatizada Mycobacteria Growth Indicator Tube (MGIT ${ }^{\circledR}$ ) 960 da Becton e Dickinson (BD), com descontaminação pelo método Petroff é um sistema comercial automatizado que utiliza o meio liquido Middlebrok 7h9 enriquecido, cujo funcionamento se dá através da fluorescência presente no tubo, que detecta a concentração de oxigênio do meio ${ }^{8,15,16}$.

A partir do cultivo com resultados positivos, são utilizados testes complementares para identificação da espécie ${ }^{17}$. 0 "fator corda" é em um teste fenotípico que detecta a presença de agregados bacilares quando realizado 0 esfregaço do cultivo, isto porque, o $M$. tuberculosis apresenta em sua parede celular o composto 6-6' Dimicolato de Trealose que quando em meio liquido forma uma película e causa agregação ${ }^{8,9,18}$. $\quad 0$ teste imunocromatográfico tuberculose Ag MPT64 TEST BIOEASY é um Teste Rápido (TR) qualitativo que identifica o CMTB a partir de um anticorpo monoclonal anti-MPT64 em combinação com culturas sólidas ou liquidas ${ }^{19}$. O Teste Rápido Molecular para Tuberculose (TRM-TB) é uma recente tecnologia laboratorial que amplifica ácidos nucleicos utilizados na detecção do CMTB e que faz a triagem de cepas resistente ao fármaco Rifampicina ${ }^{20}$. O método GeneXpert ${ }^{\circledR}$ MTB/RIF da Cepheid é um TRM-TB recomendado pela Organização Mundial da Saúde (OMS) que garante o diagnóstico da TB em 2 horas ${ }^{2,21}$. O Programa Nacional de Controle da Tuberculose (PNCT) deve estar implantado para que a detecção, acompanhamento e o isolamento dos casos sejam garantidos de forma rápida e eficiente $^{22}$. Desde setembro de 2014 o CLR-IAL-PP $\checkmark$ realiza o diagnóstico da TB pelo TRM GeneXpert $^{\circledast}$ MTB/RIF para algumas unidades prisionais. Já a cultura automatizada BACTEC MGIT ${ }^{\circledast} 960$ é realizada para todas as unidades da sua área de abrangência, desde a data de sua implantação.

Diante da importância epidemiológica da tuberculose e, sobretudo em condições favoráveis para a transmissão e propagação que as unidades prisionais representam, o presente estudo teve por objetivo, analisar a importância da cultura automatizada BACTEC MGIT ${ }^{\circledR} 960$ BD ("padrão-ouro") como forma complementar à identificação da espécie, o desempenho do TRMTB no equipamento GeneXpert ${ }^{\circledR}$ MTB/RIF e a distribuição do quantitativo da tuberculose frente à quantidade de exames realizados para o diagnóstico da tuberculose em cinco unidades prisionais da área de abrangência do Centro de Laboratório Regional - Instituto Adolfo Lutz de Presidente Prudente V (CLR IAL PP V) no período entre outubro de 2015 e outubro de 2016.

\section{METODOLOGIA}

Estudo retrospectivo avaliado e aprovado pelo Comitê de Ética em Pesquisa (CAAE n.으 50577015.6.0000.0059). Foi realizado o levantamento de dados dos exames realizados para diagnóstico da tuberculose através do sistema de Gerenciamento de Ambiente Laboratorial (GAL) no período entre outubro/2015 e outubro/2016 de amostras de pacientes oriundos de unidades prisionais de cinco municípios da área de abrangência de atendimento do CLR-IAL-PP V (A, B, C, D e E). A demonstração da população prisional abrigada, segundo a unidade estudada, o tipo de regime e a sua capacidade encontra-se na Tabela 1.

O critério da seleção de amostras a serem analisadas, foi considerar aquelas em que a quantidade de material enviado foi suficiente para diagnóstico da tuberculose, sendo possível 
processar em paralelo no GeneXpert ${ }^{\circledR}$ MTB/RIF e na cultura automatizada no BACTEC MGIT ${ }^{\oplus} 960$ da BD ou apenas no GeneXpert ${ }^{\circledR}$ MTB/RIF. Os dados foram organizados em planilha do Software Microsoft Excel ${ }^{\circledR}$. A análise estatística foi realizada segundo Ferreira e Ávila ${ }^{23}$, através de uma tabela de dupla entrada que relaciona o diagnóstico da doença e o resultado do teste, tornando possível a estimativa dos valores de sensibilidade, especificidade e valores preditivos positivos e negativos.

Tabela 1. Demonstração da população prisional, abrigada segundo a unidade estudada, o tipo de regime e a sua capacidade.

\begin{tabular}{cccc}
\hline $\begin{array}{c}\text { Unidade } \\
\text { Prisional }\end{array}$ & $\begin{array}{c}\text { Tipo de } \\
\text { Regime }\end{array}$ & Capacidade & $\begin{array}{c}\text { População } \\
\text { Prisional }\end{array}$ \\
\hline A & Fechado & 844 & 1040 \\
B & Fechado & 844 & 1512 \\
C & Fechado & 872 & 2032 \\
D & Fechado & 1247 & 2106 \\
D & Fechado & 204 & 193 \\
E & Fechado & 696 & 1408 \\
E & Semi Aberto & 247 & 455 \\
\hline
\end{tabular}

Fonte: Secretaria da Administração Penitenciária do Estado de São Paulo (http://www.sap.sp.gov.br/). Acesso em 24 de Julho de 2017.

\section{RESULTADOS}

Foram analisadas 4.509 amostras oriundas de unidades prisionais de cinco municípios da área de abrangência do CLR-IAL-PP $V$, cujos quantitativos estão distribuídos conforme a Tabela 1 (total recebido), durante o período de estudo.

Do total de amostras recebidas, apenas $36,57 \%$ (1.649) continham quantidade suficiente para realizar em paralelo a cultura automatizada e o TRM, o restante $63,43 \%$ (2.860) foram processadas apenas o TRM (Tabela 2).

Das amostras processadas em paralelo, apenas 95,2\% (1.570) permitiram obter resultados nas duas metodologias, em função da presença de contaminação em 4,79\% (79) das culturas. Comparando os resultados obtidos no GeneXpert ${ }^{\circledR}$ MTB/RIF frente ao "padrão ouro" Cultura BACTEC MGIT ${ }^{\circledR} 960$ BD (Tabela 3), obtivemos especificidade de 99,03\%, sensibilidade de $72,73 \%$ e concordância de $97 \%$ entre os testes.

A cultura no BACTEC MGIT ${ }^{\circledR} 960$ identificou 121 (7,34\%) pacientes com TB e o TRM no GeneXpert ${ }^{\circledR}$ MTB/RIF identificou 103 $(6,24 \%)$. $O$ acréscimo da cultura ao diagnóstico de TB em relação ao GeneXpert ${ }^{\circledR}$ MTB/RIF foi de 18 $(1,1 \%)$. Para este acréscimo, foram realizados testes complementares de identificação de espécies ("fator corda", TR e PCR no IAL Central), e destas, 6 (33,3\%) apresentaram contaminação não sendo possível realizar a identificação da espécie, 1 (5,6\%) não houve crescimento de BAAR, $1(5,6 \%)$ apresentou cultura mista de MTB e MNT; e em 10 (55,5\%) foi identificado crescimento de MNTs (Mycobacterium avium; Mycobacterium chelonae; Mycobacterium nebrakense; Mycobacterium abcessus; Mycobacterium intracellulare e Mycobacterium $s p$.). A distribuição de resultados por unidade prisional nas diferentes metodologias está apresentada na Tabela 4. 
Tabela 2. Quantidade de exames realizados em paralelo no TR GeneXpert ${ }^{\circledR}$ MTB/RIF e na Cultura BACTEC MGIT $^{\oplus} 960$ BD e apenas no GeneXpert ${ }^{\circledR}$ MTB/RIF.

\begin{tabular}{lcccccc}
\hline & \multicolumn{7}{c}{ Unidade Prisional } \\
\hline Teste / Resultado & A & B & C & D & E & Total \\
\hline $\begin{array}{l}\text { TR GeneXpert MTB/RIF em paralelo com } \\
\text { Cultura BACTEC MGIT® } 960 \text { BD }\end{array}$ & 160 & 560 & 419 & 248 & 262 & $\begin{array}{c}1649 \\
(36,57 \%)\end{array}$ \\
GeneXpert ${ }^{\circledR}$ MTB/RIF & 228 & 801 & 745 & 276 & 810 & $\begin{array}{c}2860 \\
(63,43 \%)\end{array}$ \\
Total Recebido & $\mathbf{3 8 8}$ & $\mathbf{1 3 6 1}$ & $\mathbf{1 1 6 4}$ & $\mathbf{5 2 4}$ & $\mathbf{1 0 7 2}$ & $\mathbf{4 5 0 9}$ \\
& $\mathbf{( 8 , 6 1 \% )}$ & $\mathbf{( 3 0 , 1 8 \% )}$ & $\mathbf{( 2 5 , 8 1 \% )}$ & $\mathbf{( 1 1 , 6 2 \% )}$ & $\mathbf{( 2 3 , 7 8 \% )}$ & $\mathbf{( 1 0 0 \% )}$ \\
\hline
\end{tabular}

Fonte: Gerenciador de Ambiente Laboratorial (GAL) e CLR-IAL-PP V.

Tabela 3. Tabela de dupla entrada, demonstrando o desempenho entre os resultados apresentados no TRM GeneXpert ${ }^{\circledR}$ MTB/RIF, frente ao "padrão ouro" obtidos na Cultura BACTEC MGIT 960 BD.

\section{CULTURA MGIT 960 BD}

\begin{tabular}{cccccc}
\hline TRM & & Positiva & Negativa & Contaminada & TOTAL \\
GENEXPERT $^{\circledR}$ & Detectado & $88(5,34 \%)$ & $14(0,85 \%)$ & $1(0,06 \%)$ & $103(6,24 \%)$ \\
MTB/RIF & Não detectado & $33(2,0 \%)$ & $1435(87,02 \%)$ & $78(4,73 \%)$ & $1546(93,76 \%)$ \\
& TOTAL & $121(7,34 \%)$ & $1449(87,88 \%)$ & $79(4,79 \%)$ & $1649(100 \%)$ \\
\hline
\end{tabular}

Fonte: Gerenciador de Ambiente Laboratorial (GAL) e CLR-IAL-PP V.

Tabela 4. Distribuição de resultados por unidade prisional nas diferentes metodologias.

\begin{tabular}{|c|c|c|c|c|c|c|c|}
\hline \multirow{2}{*}{\multicolumn{2}{|c|}{ Teste / Resultado }} & \multicolumn{6}{|c|}{ Unidade Prisional } \\
\hline & & A & B & C & D & $E$ & TOTAL \\
\hline \multirow{3}{*}{$\begin{array}{c}\text { TRM } \\
\text { GeneXpert } \\
\text { MTB/RIF }\end{array}$} & Detectável & 8 & 51 & 54 & 28 & 37 & $178(3,94 \%)$ \\
\hline & NãoDetectável & 380 & 1310 & 1110 & 496 & 1035 & $4331(96,06 \%)$ \\
\hline & Total & $\begin{array}{c}388 \\
(8,61 \%)\end{array}$ & $\begin{array}{c}1361 \\
(30,18 \%)\end{array}$ & $\begin{array}{c}1164 \\
(25,81 \%)\end{array}$ & $\begin{array}{c}524 \\
(11,62 \%)\end{array}$ & $\begin{array}{c}1072 \\
(23,78 \%)\end{array}$ & $4509(100 \%)$ \\
\hline \multirow{4}{*}{$\begin{array}{c}\text { Cultura } \\
\text { BACTEC } \\
\text { MGIT }^{\circledR} 960 \\
\text { BD }\end{array}$} & Positiva & 4 & 41 & 32 & 22 & 22 & $121(7,34 \%)$ \\
\hline & Negativa & 151 & 500 & 366 & 217 & 215 & $1449(87,88 \%)$ \\
\hline & Contaminada & 5 & 19 & 21 & 9 & 25 & 79 (4,79\%) \\
\hline & Total & $\begin{array}{c}160 \\
(9,71 \%)\end{array}$ & $\begin{array}{c}560 \\
(33,96 \%)\end{array}$ & $\begin{array}{c}419 \\
(25,41 \%)\end{array}$ & $\begin{array}{c}248 \\
(15,04 \%)\end{array}$ & $\begin{array}{c}262 \\
(15,88 \%)\end{array}$ & $1649(100 \%)$ \\
\hline
\end{tabular}

Fonte: Gerenciador de Ambiente Laboratorial (GAL) e CLR-IAL-PP V.

\section{DISCUSSÃO}

A quantidade de amostras recebidas (4.509) de cinco unidades prisionais atendidas pelo CLR-IAL-PP V, foi superior ao estudo realizado por Sappuci et $\mathrm{al}^{24}$ quando investigou o quantitativo (2.761) recebido de outubro/2015 a outubro/2016 da mesma área de atendimento, ainda que tenha sido analisado amostras de apenas quatro das cinco unidades do presente estudo. Além disso, o quantitativo da população carcerária (Tabela 1) abrigadas nas unidades prisionais, demonstra ser necessário melhoria em relação à vigilância em saúde, visto a quantidade de amostras recebidas para diagnóstico ser pequena, quando comparada a população carcerária existente. Tal fato é coadjuvante por delinear a importância do diagnóstico da tuberculose na população privada de liberdade, uma vez que está aumentada consideravelmente. A Organização Mundial da Saúde ${ }^{25}$, alerta que o atraso no diagnóstico e isolamento do paciente com tuberculose, intensifica a cadeia de transmissão deste agravo.

De acordo com o exposto na Figura 1, a unidade prisional que enviou maior quantidade foi a unidade "B", seguida da "C", " $E$ ", " $D$ ", e "A", entretanto a unidade prisional que apresentou a maior quantidade de resultados positivos (Tabela 
4), tanto no TRM quanto na cultura foi a unidade "C", seguida da "B", "E", "D" e "A". Do total recebido, apenas 36,57 (1.649) continham quantidade suficiente para processar em paralelo a cultura automatizada e o TRM. Os dados da Tabela 2 nos permitem observar a péssima qualidade dos materiais biológicos enviados, uma vez que eram insuficientes ou inviáveis. Esses dados apontam a necessidade de melhorias, nas instruções para coleta e conferência de amostras, no transporte e agilidade para que cheguem até o local de análise com qualidade.

Ainda que tenha ocorrido contaminação de culturas no presente estudo, os valores encontrados estão dentro do que $\circ \mathrm{MS}^{8}$ preconiza, que é um índice entre 3 e $5 \%$. Já as $2.860(63,43 \%)$ amostras que foram insuficientes para ser realizado em paralelo nas duas metodologias, tiveram resultados apenas no TRM GeneXpert MTB/RIF, isso porque o diagnóstico com esta metodologia é mais ágil. Atualmente, os processos de preparo da amostra já possibilitam que o TRM possa ser realizado com o sedimento do preparo da cultura, assim é garantido que todas as amostras sejam processadas em paralelo, resultando em maior eficácia e segurança no diagnóstico da TB.

A análise dos testes em paralelo, possibilitou a avaliação da sensibilidade $e$ especificidade do GeneXpert MTB/RIF demostrando um bom desempenho da nova tecnologia frente ao "padrão-ouro". Entretanto, a cultura possibilitou um acréscimo de $1,1 \%$ ao diagnóstico em relação ao TRM (Tabela 3), permitindo a realização de exame complementar para identificação da espécie. Tal fato, acoplado ao resultado de exames complementares realizados nas amostras positivadas apenas na cultura, demonstra quão importante é a realização do cultivo na detecção da infecção, tanto do CMTB quanto de MNT com sintomatologia clínica semelhante, tendo em vista a detecção apenas do CMTB no GeneXpert ${ }^{\circledast}$ MTB/RIF. Resultados semelhantes foram encontrados em estudos anteriores realizados por Sappuci et $\mathrm{al}^{24}$, no entanto o acréscimo da cultura ao diagnóstico foi menor $(0,25 \%)$. 0 fato de ocorrer amostras positivas para tuberculose, apenas no TRM $(0,85 \%)$, pode ser explicado pela inviabilidade de crescimento do bacilo na cultura, sejam por inativação em caso de uso de medicamentos, más condições de armazenamento, transporte e/ou tempo de coleta.

\section{CONCLUSÃO}

Os dados aqui apresentados, nos permitem avaliar qual unidade prisional teve maior incidência de positivos e detectáveis para TB. Foi apontada a necessidade de melhoria da vigilância em saúde, com recapacitação das equipes de saúde das unidades quanto a qualidade e quantidade das amostras coletadas, para assegurar a qualidade do diagnóstico realizado. É de suma importância avaliar a taxa de ocupação frente a capacidade populacional das unidades prisionais, para o entendimento da situação epidemiológica deste agravo. O sistema GeneXpert ${ }^{\circledR}$ MTB/RIF é uma ferramenta de grande utilidade para o PNCT, possibilitando agilidade ao diagnóstico e no tratamento precoce contribuindo para ruptura na cadeia de transmissão desta doença. Todavia a cultura é de suma importância e deve ser realizada, como forma complementar à identificação da espécie e do tipo de infecção.

\section{AGRADECIMENTOS}

Ao patrocinador principal, o Ministério da Saúde, e ao Instituto Adolfo Lutz.

\section{CONFLITO DE INTERESSES}

Os autores declararam não haver qualquer potencial conflito de interesse que possa interferir na imparcialidade deste trabalho cientifico.

\section{REFERÊNCIAS}

1. Sakamoto K. The pathology of Mycobacterium tuberculosis infection. Vet Pathol. 2012;49(3):423-39. DOI: https://doi.org/10.1177/0300985811429313

2. Boletim Brasileiro de Avaliação Tecnológica em Saúde. $\quad \mathrm{XPERT}^{\circledR} \mathrm{MTB} / \mathrm{RIF}$ no Diagnóstico da Tuberculose Pulmonar. Brasil: ANVISA. 2011.

3. Antunes A, Viveiros F, Carvalho A, Duarte R. Micobacterioses não-tuberculosas - das manifestações clínicas ao tratamento. Arq Med. 2012;26(1):25-30.

4. Abrahão RMCM. Diagnóstico da tuberculose na população carcerária dos distritos policiais da zona oeste da cidade de São Paulo. [Tese de doutorado]. São Paulo: Faculdade de Saúde Pública, 2004. DOI: https://doi.org/10.11606/T.6.2004.tde-03032011$\underline{144652}$ 
5. Oliveira HB, Cardoso JC. Tuberculose no sistema prisional de Campinas, São Paulo, Brasil. Rev Panam Salud Publica. 2004;15(3):194-9, DOI: https://doi.org/10.1590/S1020-49892004000300008

6. Delogu G, Sali M, Fadda G. The biology of Mycobacterium Tuberculosis infection. Mediterr J Hematol Infect Dis. 2013;5(1):e2013070. DOI: https://doi.org/10.4084/mjhid.2013.070

7. Palomino JC. Nonconventional and new methods in the diagnosis of tuberculosis: feasibility and applicability in the field. Eur Respir J. 2005;26(2):33950. DOI:

https://doi.org/10.1183/09031936.05.00050305

8. Ministério da Saúde. Secretaria de Vigilância em Saúde. Departamento de Vigilância Epidemiológica. Manual nacional de vigilância laboratorial da tuberculose e outras micobactérias. Brasília, 2008.

9. Ministério da Saúde. Secretaria de Vigilância em Saúde. Tuberculose: Diagnóstico Laboratorial Baciloscopia. Manual TELELAB. Brasília, 2001.

10. Ferri AO, Aguiar B, Wilhelm CM, Schmidt D, Fussieger F, Picoli SU. Diagnóstico da tuberculose: uma revisão. Rev Liberato. 2014;15(24):105-212.

11. Kennedy DH, Fallon RJ. Tuberculosis diagnostic. J Am Med Assoc. 1979;241:264-8. DOI: https://doi.org/10.1001/jama.1979.03290290032021

12. Abrahão RMCM. Tuberculose humana causada pelo Mycobacterium bovis: considerações gerais e a importância dos reservatórios animais. Arch Vet Scienc. 1999;4(1):5-15.

13. Ryu YJ. Diagnosis of pulmonary tuberculosis: recent advances and diagnostic. Tuberc Respir Dis (Seoul). 2015;78(2):64-71. DOI: https://doi.org/10.4046/trd.2015.78.2.64

14. Ministério da Saúde. Secretaria de Vigilância em Saúde. Departamento de Vigilância Epidemiológica. Manual de Bacteriologia da Tuberculose. 3.ed. Rio de Janeiro, 2005.

15. Hanna BA, Ebrahimzadeh A, Elliott LB, Morgan MA, Novak SM, Rusch-Gerdes S. Multicenter evaluation of the BACTEC MGIT 960 system for recovery of mycobacteria. J Clin Microbiol. 1999;37:748-52.

16. Almeida EA, Santos MAA, Afiune JB, Spada DTA, Melo, FAF. Rendimento da cultura de escarro na comparação de um sistema de diagnóstico automatizado com o meio de Lowenstein-Jensen para o diagnóstico da tuberculose pulmonar. J Bras
Pneumol.

2005;31(3):231-6.

DOI:

https://doi.org/10.1590/S1806-37132005000300009

17. Santos MO. Micobactérias: identificação e perfil de sensibilidade a tuberculostáticos em amostras isoladas no laboratório central de saúde pública do estado do Piauí, janeiro 2014 a março de 2015. [Tese de doutorado]. Teresina, PI: Fundação Oswaldo Cruz, 2015.

18. Simeão FCS, Chimara E, Oliveira RS, Yamauchi U, Latrilha FO, Telles MAS. Detecção do fator corda e avaliação do aspecto macroscópico das colônias de micobactérias: um eficiente teste de triagem combinado para a identificação presuntiva do complexo Mycobacterium tuberculosis em meios sólidos. J Bras Pneumol. 2009;35(12):1212-6.

19. Alere, TB Ag MPT64 TEST BIOEASY. [bula]. São Paulo, 2013.220.

20. Lemos AC, Matos ED. Multidrug-resistant tuberculosis. Braz J Infect Dis. 2013;17(2):239-46. DOI: https://doi.org/10.1016/j.bjid.2013.01.007

21. World Health Organization. Roadmap for rolling Out Xpert MTB/RIF for rapid diagnosis of TB and MDRTB. Geneva, 2010

22. Oliveira LGD, Natal S, Camacho LAB. Análise da implantação do Programa de Controle da Tuberculose em unidades prisionais no Brasil. Cad Saude Publica. 2015;31:543554, DOI: https://doi.org/10.1590/0102$\underline{311 \times 00042914}$

23. Ferreira AW, Ávila SLM. Diagnóstico laboratorial das principais doenças infecciosas e auto-imunes. 2.ed. Rio de Janeiro: Guanabara-Koogan; 2001.

24. Sappuci FHF, D' Andrea LAZ, Saeki, EK, Lima, PES, Alfredo MX, Mizusaki, S, Silva, MCMF, Romão MM. Pulmonary tuberculosis in prisions of the western São Paulo state between 2014 and 2015. Anais do $5^{\circ}$ Simpósio Internacional Microbiologia Clínica; 13-15 mai 2016; São Pedro. São Paulo, 2016.

25. World Health Organization. Tuberculosis in prisons. Geneva, 2013.

Recebido para publicação em 09/11/2016

Revisado em 22/03/2018

Aceito em 10/05/2018 